\title{
Violence risk assessment and treatment
}

\author{
Katherine D. Warburton, ${ }^{1 *}$ and Charles L. Scott ${ }^{2}$
}

\footnotetext{
${ }^{1}$ California Department of State Hospitals, Sacramento, California, USA

${ }^{2}$ University of California, Davis Medical Center, Sacramento, California, USA
}

Violence in inpatient psychiatric settings is a serious problem and it is not going away. Each year, psychiatric patients physically injure nearly 1 in 4 psychiatric nurses. ${ }^{1,2}$ The damage done from these injuries is not limited to the physical and emotional harm caused to the individual mental health provider. Quite the contrarypoorly managed aggression committed by psychiatric patients results in a treating environment characterized by fear and anxiety for both providers and patients, a decreased ability to provide effective care, and a draining of limited financial resources to address the medical and legal consequences of patients' violent behavior. Something more must be done.

The relationship between violence and psychiatric illness is complex and controversial. This Special Issue provides a comprehensive approach to conceptualizing, assessing, and treating inpatient aggression. In an opinion piece, Warburton explores the possibility that the complexion of public psychiatric populations is changing, and suggests that diagnostic methodologies and treatment approaches may need to change as well.

The multifactorial nature of violence necessitates exploration from multiple perspectives, ranging from the biological to the philosophical. Providing an important neurobiological foundation, Pardini et al have contributed original research that suggests that lesion location modulates the relationship between penetrating traumatic brain injury-related aggression and the dopaminergic system; the article proposes possible therapeutic strategies based on those findings.

Multiple contributions explore the science, utility, and limitations of violence risk assessment approaches and instruments. In their article, Monahan and Skeem provide an important overview of the history of violence risk assessment with clearly defined descriptions of

* Address for correspondence: Katherine Warburton, California Department of State Hospitals, 1600 9th Street, Suite 400, Sacramento, CA 95814, USA.

(Email: katherine.warburton@dsh.ca.gov) various violence risk assessment approaches. Scott and Resnick address the clinical assessment of specific psychotic and mood disorder symptoms relevant to evaluating a patient's risk of future dangerousness. In their article, McDermott and Holoyda build on the foundation of violence risk assessment by describing the importance of understanding recent research that describes a typology of aggressive incidents, allowing providers to gain insight into assault precipitants and matched interventions to reduce such aggression.

Several articles on psychopharmacology explore options for treating violence when standard therapies based on published guidelines fail. Morrissette and Stahl conduct a review of when and how to utilize the higher risk practices of treating aggression with high-dose monotherapy and polypharmacy. Brown et al of the Broadmoor group provide a promising case series on the treatment of anti-social aggression with clozapine. Similarly, Hotham et al, also from the Broadmoor group, contribute a separate case series showing some success augmenting clozapine with amisulpride to reduce aggression in forensic psychiatric patients. Additionally, Meyer provides principles and evidence for high plasma antipsychotic prescribing in treatment resistant violent forensic patients.

Citrome and Volavka provide a comprehensive literature review, emphasizing the important distinction between acute and preventative management of violence, and providing evidence-based strategies for both. Volavka et al also conducted an examination of Clinical Antipsychotic Trials of Intervention Effectiveness (CATIE) data to analyze anti-hostility effect, and found a difference between treatments. Treatment implications of those findings are discussed. Finally, Stahl et al provide comprehensive guidelines for the assessment and treatment of violence.

Inpatient psychiatric aggression is multifactorial and complicated. Beyond the obvious toll on the health of victims, there are additional costs related to the disruption of treatment delivery. It is important to acknowledge and address the issue, as well as to apply established 
principles when conceptualizing, assessing, and treating aggression.

\section{Disclosures}

Katherine Warburton does not have anything to disclose. Charles Scott does not have anything to disclose.
REFERENCES:

1. Carmel H, Hunter M. Staff injuries from inpatient violence. Hosp Community Psychiatry 1989; 40(1): 41-46.

2. Hunter M, Carmel H. The cost of staff injuries from inpatient violence. Hosp Community Psychiatry. 1992; 43(6): 586-588. 